\title{
Cardiac magnetic resonance imaging characteristics for the differentiation of athlete's heart from inherited cardiomyopathies
}

\author{
Benedikt Bernhard ${ }^{1} \cdot$ Babken Asatryan $^{1} \cdot$ Christoph Gräni $^{1}$
}

Published online: 29 June 2021

(c) The Author(s), under exclusive licence to Springer Nature B.V. 2021

Hypertrophic cardiomyopathy (HCM), dilated cardiomyopathy (DCM) and arrhythmogenic cardiomyopathy (ACM) represent a group of inherited cardiomyopathies with highly variable phenotypic features [1]. Especially in early stages of the disease, an asymptomatic course is common, often accompanied with incidental diagnosis during routine clinical examination. Myocardial alterations may lead to left-, right- or biventricular structural and/or functional abnormalities, especially in the advanced stages of the disease and potential consecutive life-threatening ventricular arrhythmias with sudden cardiac death (SCD) [1-3]. However, a sizeable proportion of SCD occurs in previously asymptomatic, apparently healthy individuals, underlining the need for early diagnosis, adequate risk-stratification and correct indication for implantable cardioverter defibrillator (ICD) [4].

Over the past two decades, advances in noninvasive imaging and genetic sequencing have contributed importantly to our understanding of cardiomyopathies, and resulted in increased recognition of these potentially life-threatening but manageable conditions and their remarkable prevalence, conservatively estimated to be as high as $1 / 250$ for DCM, $1 / 500$ for HCM and 1/2000-1/5000 for ACM [5-8]. In addition, phenotypic mimics previously misdiagnosed as an inherited cardiomyopathy have been increasingly recognized in parallel to the implementation of more high-resolution imaging technologies. Nevertheless, diagnosis remains challenging due to the lack of specific clinical, electrocardiographic and imaging characteristics. Whilst genetic testing

This comment refers to the article available online at https://doi. org/10.1007/s 10554-021-02280-6

$\triangle$ Christoph Gräni

christoph.graeni@insel.ch

1 Department of Cardiology, Inselspital, Bern University Hospital, University of Bern, CH-3010 Bern, Switzerland helps in identifying asymptomatic family members of individuals confirmed to have a genetic cardiomyopathies, the incomplete penetrance, variable disease expressivity and to-date partial knowledge of the genetic architecture of cardiomyopathies are some of the challenges limiting the use of genetic testing for primary diagnosis [9]. In this setting, cardiac magnetic resonance imaging (CMR) has emerged as a key tool for the differential diagnosis of cardiomyopathies thanks to its high spatial resolution and excellent soft-tissue characterization [10]. However, inherited cardiomyopathies can show CMR features resembling non-pathological structural changes of the myocardium. This is particularly the case in exercise-induced cardiac remodeling, often referred to as 'athlete's heart' (AH). Repetitive intense exercise, the core to the increasingly enhanced athletic activities, can result in long-term remodeling of the cardiovascular system and increases in cardiac dimensions and cardiac mass [11]. Male sex, endurance sports and higher training volumes were found to be associated with the extent of adaptions [12]. Endurance sports (e.g. cycling, long-distance running), associated with a volume overload, predominantly impact cavity sizes, while static sports (e.g. weight lifting), associated with pressure overload, have a disproportional higher impact on left ventricular (LV) wall thickness [13]. Structural, electrical and functional changes often include both ventricles and the atria, and AH may mimic DCM, HCM, and rarely ACM.

An accurate and timely delineation among these overlapping phenotypes is central to the patient's management. While in HCM and ACM, exercise restriction is part of SCD prevention strategy; this can have severe and devastating impact on the career and quality of life in athletes incorrectly diagnosed with a heritable cardiomyopathy. Of note, the need for exercise restriction in patients with HCM is discussed controversially with recent evidence indicating that systematic exercise restriction is not fully justified [14-17]. In ACM families, cessation of competitive exercise activities 
remains crucial not only for prevention of SCD in probands, but also in asymptomatic, pathogenic variant-carrier family members [14, 18]. Endurance sports can not only lead to features mimicking ACM, but also trigger the progression from carrier status to penetrant disease fulfilling the structural and electrical criteria of ACM, with ensuing increased arrhythmic risk $[18,19]$. Data on the effect of static sport restriction is scarce [20].

Apart from its therapeutic considerations, sports restriction can also be a part of the diagnostic approach. Reversible LV hypertrophy after detraining, indicate an $\mathrm{AH}$ and makes the presence of HCM less likely [21]. Despite from the lack of evidence for a generalization of this approach, de-training elite athletes also impacts their career, underlining the need for precise non-invasive diagnostic modalities. On the other hand, missing the diagnosis of an inherited cardiomyopathy can delay appropriate prevention measures and therapy, in the worst-case scenario exposing the patient to the occurrence of a malignant ventricular tachyarrhythmia without ICD-protection.

In this context, Kübler et al. [22] aimed to identify imaging features that allow distinguishing $\mathrm{AH}$ from inherited cardiomyopathies by comparing CMR characteristics of patients with DCM, ACM and HCM and individuals with $\mathrm{AH}$ [22]. We congratulate the authors on this important study, that included a systematic analysis of 40 athletes and patients suffering from DCM $(n=48)$, ACM $(\mathrm{n}=18)$ and HCM $(\mathrm{n}=14)$. In line with previous studies, the authors were able to highlight several overlapping, as well as delineating features among the various entities. DCM was characterized by severely impaired LV ejection fraction (LVEF), and LV dilatation, while LVEF and LV end diastolic volume index (LVEDVI) were only slightly reduced in $\mathrm{AH}$. Athletes showed reduced right ventricular EF (RVEF), RV enlargement and elevated LV myocardial mass. In contrast, HCM patients showed increased interventricular septum thickness and LV remodeling index, but lower RV myocardial thickness. In accordance with the recent knowledge establishing ACM as a mostly biventricular disease [20], the authors identified reduced LVEF, reduced LV stroke volume index (LVSVI), presence of LV late gadolinium enhancement (LGE) and LV wall motion abnormalities in patients with ACM. LGE showed high utility in delineating physiological remodeling from pathological alterations. Whilst it was only present in one patient with AH (5\%), evidence for LGE was found in $57 \%$ of patients with $\mathrm{HCM}, 56 \%$ with ACM and $44 \%$ with DCM. Other studies have also found an association between LGE and outcomes in DCM [23], HCM [24], and ACM [25], underlining the prognostic value of this marker in inherited cardiomyopathies. As mentioned by the authors, LGE can be considered as a pathologic finding, indicating fibrotic changes of the myocardium.
Incidental findings of scar can indicate a 'healed' disease, such as myocarditis [26], ischemia [27], or corrected structural heart disease, as may be the case in the one athlete in whom Kübler et al. found LGE. Etiologies can be further defined according to the LGE distribution and localization. Similarly, LV and/or RV wall motion abnormalities such as hypo- and akinesia are often identified in all cardiomyopathy subtypes but, as shown in this study, are not seen in AH. Concomitant proportional enlargement of all cavities in the absence of other structural or functional abnormalities can be considered as a sign of AH. This form of remodeling allows for increased stroke volumes and hence higher cardiac output in athletes.

Strengths of the presented study are the multicenter design with a predefined image acquisition approach and the well-defined patient population with histologic confirmation of the diagnosis in the majority of participants. The study is limited to patients with pathological findings with an unequivocal definitive diagnosis and signs of advanced disease and elite athletes whose fitness can be expected to be in the highest percentile of the exercising general population, hence the study prevents drawing stronger conclusions. Furthermore, mostly endurance athletes were included while static sports were under-represented. Delineation between athlete's heart, DCM, ACM and HCM will remain a challenge in patients with less clear diagnosis and overlapping phenotypes first presenting with unknown conditions. Prospective studies investigating such cases in the intermediate zone could help better define features distinguishing $\mathrm{AH}$ from early stages of inherited cardiomyopathies.

Besides the CMR characteristics identified by Kübler et al., previous work in this field found functional cardiac evaluation during exercise and parametric mapping, including extracellular volume (ECV) fraction to be useful in differentiating $\mathrm{AH}$ from structural heart disease [28, 29]. ECV has been shown to decline in individuals exposed to repetitive exercise since the cellular compartment expands with hypertrophy of myocytes [30]. On the contrary, ECV increases in several cardiomyopathy subtypes, thus it should also be taken into account in unclear cases. With regard to reference values, a recent meta-analysis including 983 athletes provides "normal ranges" on the distribution of LV/RV dimensions and LVEF in individuals classified as "healthy" and could help to define the maximum limit of cardiac adaptations estimated to be physiologic [11].

In conclusion, detailed analysis of CMR features can help to distinguish physiologic adaptation in elite athletes from inherited cardiomyopathies. Nevertheless, diagnosis cannot be made solely on imaging findings and all information on clinical presentation, personal and family history, and electrocardiogram findings should be implemented in differentiating athlete's heart from inherited cardiomyopathies. 


\section{Declarations}

Conflict of interest The authors have no conflict of interest to declare.

\section{References}

1. Elliott P, Andersson B, Arbustini E, Bilinska Z, Cecchi F, Charron $P$ et al (2008) Classification of the cardiomyopathies: a position statement from the European society of cardiology working group on myocardial and pericardial diseases. Eur Heart $\mathrm{J}$ 29(2):270-276

2. Asatryan B, Vital C, Kellerhals C, Medeiros-Domingo A, Gräni C, Trachsel LD et al (2017) Sports-related sudden cardiac deaths in the young population of Switzerland. PLoS ONE 12(3):e0174434

3. Gräni C, Chappex N, Fracasso T, Vital C, Kellerhals C, Schmied $\mathrm{C}$ et al (2016) Sports-related sudden cardiac death in Switzerland classified by static and dynamic components of exercise. Eur J Prev Cardiol 23(11):1228-1236

4. Al-Khatib SM, Stevenson WG, Ackerman MJ, Bryant WJ, Callans DJ, Curtis AB et al (2018) 2017 AHA/ACC/HRS guideline for management of patients with ventricular arrhythmias and the prevention of sudden cardiac death: executive summary: a report of the American college of cardiology/American heart association task force on clinical practice guidelines and the heart rhythm society. J Am Coll Cardiol 72(14):1677-1749

5. McKenna WJ, Judge DP (2021) Epidemiology of the inherited cardiomyopathies. Nat Rev Cardiol 18(1):22-36

6. Ingles J, Goldstein J, Thaxton C, Caleshu C, Corty EW, Crowley SB et al (2019) Evaluating the clinical validity of hypertrophic cardiomyopathy genes. Circ Genom Precis Med 12(2):e002460

7. James CA, Jongbloed JDH, Hershberger RE, Morales A, Judge DP, Syrris P et al (2021) An international evidence based reappraisal of genes associated with arrhythmogenic right ventricular cardiomyopathy (ARVC) using the ClinGen framework. Circ Genom Precis Med. https://doi.org/10.1161/CIRCGEN.120. 003273

8. Jordan E, Peterson L, Ai T, Asatryan B, Bronicki L, Brown E et al (2021) An evidence-based assessment of genes in dilated cardiomyopathy. Circulation. https://doi.org/10.1161/CIRCULATIO NAHA. 120.053033

9. Marian AJ, Asatryan B, Wehrens XHT (2020) Genetic basis and molecular biology of cardiac arrhythmias in cardiomyopathies. Cardiovasc Res 116(9):1600-1619

10. Patel AR, Kramer CM (2017) Role of cardiac magnetic resonance in the diagnosis and prognosis of nonischemic cardiomyopathy. JACC Cardiovasc Imaging 10(10):1180-93

11. D'Ascenzi F, Anselmi F, Piu P, Fiorentini C, Carbone SF, Volterrani L et al (2019) Cardiac magnetic resonance normal reference values of biventricular size and function in male athlete's heart. JACC Cardiovasc Imaging 12(9):1755-1765

12. Csecs I, Czimbalmos C, Toth A, Dohy Z, Suhai IF, Szabo L et al (2020) The impact of sex, age and training on biventricular cardiac adaptation in healthy adult and adolescent athletes: cardiac magnetic resonance imaging study. Eur J Prev Cardiol 27(5):540-549

13. Pelliccia A, Maron MS, Maron BJ (2012) Assessment of left ventricular hypertrophy in a trained athlete: differential diagnosis of physiologic athlete's heart from pathologic hypertrophy. Prog Cardiovasc Dis 54(5):387-396

14. Pelliccia A, Sharma S, Gati S, Bäck M, Börjesson M, Caselli S et al (2021) 2020 ESC guidelines on sports cardiology and exercise in patients with cardiovascular disease. Eur Heart $\mathbf{J}$ 42(1):17-96
15. Pelliccia A, Fagard R, Bjørnstad HH, Anastassakis A, Arbustini E, Assanelli D et al (2005) Recommendations for competitive sports participation in athletes with cardiovascular disease: a consensus document from the study group of sports cardiology of the working group of cardiac rehabilitation and exercise physiology and the working group of myocardial and pericardial diseases of the European society of cardiology. Eur Heart J 26(14):1422-1445

16. Lampert R, Olshansky B, Heidbuchel H, Lawless C, Saarel E, Ackerman M et al (2017) Safety of sports for athletes with implantable cardioverter-defibrillators: long-term results of a prospective multinational registry. Circulation 135(23):2310-2312

17. Pelliccia A, Lemme E, Maestrini V, Di Paolo FM, Pisicchio C, Di Gioia G et al (2018) Does sport participation worsen the clinical course of hypertrophic cardiomyopathy? Clinical outcome of hypertrophic cardiomyopathy in athletes. Circulation 137(5):531-533

18. James CA, Bhonsale A, Tichnell C, Murray B, Russell SD, Tandri $\mathrm{H}$ et al (2013) Exercise increases age-related penetrance and arrhythmic risk in arrhythmogenic right ventricular dysplasia/ cardiomyopathy-associated desmosomal mutation carriers. J Am Coll Cardiol 62(14):1290-1297

19. La Gerche A, Robberecht C, Kuiperi C, Nuyens D, Willems R, de Ravel $\mathrm{T}$ et al (2010) Lower than expected desmosomal gene mutation prevalence in endurance athletes with complex ventricular arrhythmias of right ventricular origin. Heart (British Cardiac Society) 96(16):1268-1274

20. Towbin JA, McKenna WJ, Abrams DJ, Ackerman MJ, Calkins H, Darrieux FCC et al (2019) 2019 HRS expert consensus statement on evaluation, risk stratification, and management of arrhythmogenic cardiomyopathy: executive summary. Heart Rhythm 16(11):e373-e407

21. Maron BJ, Pelliccia A, Spataro A, Granata M (1993) Reduction in left ventricular wall thickness after deconditioning in highly trained olympic athletes. Br Heart J 69(2):125-128

22. Kübler J, Burgstahler C, Brendel JM, Gassenmaier S, Hagen $\mathrm{F}$, Klingel $\mathrm{K}$ et al (2021) Cardiac MRI findings to differentiate athlete's heart from hypertrophic (HCM), arrhythmogenic right ventricular (ARVC) and dilated (DCM) cardiomyopathy. Int J Cardiovasc Imaging. https://doi.org/10.1007/s10554-021-02280-6

23. Becker MAJ, Cornel JH, van de Ven PM, van Rossum AC, Allaart CP, Germans T (2018) The prognostic value of late gadoliniumenhanced cardiac magnetic resonance imaging in nonischemic dilated cardiomyopathy: a review and meta-analysis. JACC Cardiovasc Imaging 11(9):1274-1284

24. Green JJ, Berger JS, Kramer CM, Salerno M (2012) Prognostic value of late gadolinium enhancement in clinical outcomes for hypertrophic cardiomyopathy. JACC Cardiovasc Imaging 5(4):370-377

25. Aquaro GD, Pingitore A, Di Bella G, Piaggi P, Gaeta R, Grigoratos $C$ et al (2018) Prognostic role of cardiac magnetic resonance in arrhythmogenic right ventricular cardiomyopathy. Am J Cardiol 122(10): 1745-1753

26. Eichhorn C, Bière L, Schnell F, Schmied C, Wilhelm M, Kwong RY et al (2020) Myocarditis in athletes is a challenge: diagnosis, risk stratification, and uncertainties. JACC Cardiovasc Imaging 13(2 Pt 1):494-507

27. Gräni C, Benz DC, Gupta S, Windecker S, Kwong RY (2020) Sudden cardiac death in ischemic heart disease: from imaging arrhythmogenic substrate to guiding therapies. JACC Cardiovasc Imaging 13(10):2223-2238

28. Claessen G, Schnell F, Bogaert J, Claeys M, Pattyn N, De Buck F et al (2018) Exercise cardiac magnetic resonance to differentiate athlete's heart from structural heart disease. Eur Heart J Cardiovasc Imaging 19(9):1062-1070

29. Mordi I, Carrick D, Bezerra H, Tzemos N (2016) T1 and T2 mapping for early diagnosis of dilated non-ischaemic cardiomyopathy 
in middle-aged patients and differentiation from normal physiological adaptation. Eur Heart J Cardiovasc Imaging 17(7):797-803

30. McDiarmid AK, Swoboda PP, Erhayiem B, Lancaster RE, Lyall GK, Broadbent DA et al (2016) Athletic cardiac adaptation in males is a consequence of elevated myocyte mass. Circ Cardiovasc Imaging 9(4):e003579
Publisher's Note Springer Nature remains neutral with regard to jurisdictional claims in published maps and institutional affiliations. 